\title{
Alter
}

Revue de phénoménologie

$28 \mid 2020$

La religion

\section{La mort de Dieu. Sartre versus Heidegger}

\section{Philippe Cabestan}

\section{(2) OpenEdition}

Journals

Édition électronique

URL : https://journals.openedition.org/alter/1991

DOI : 10.4000/alter.1991

ISSN : 2558-7927

\section{Éditeur :}

Association ALTER, Archives Husserl (CNRS-UMR 8547)

\section{Édition imprimée}

Date de publication : 1 novembre 2020

Pagination : 25-39

ISBN : 978-2-9550449-6-4

ISSN : 1249-8947

\section{Référence électronique}

Philippe Cabestan, «La mort de Dieu. Sartre versus Heidegger », Alter [En ligne], 28 | 2020, mis en ligne le 22 décembre 2020, consulté le 14 octobre 2021. URL : http://journals.openedition.org/alter/1991 ; DOI : https://doi.org/10.4000/alter.1991

Ce document a été généré automatiquement le 14 octobre 2021

Revue Alter 


\title{
La mort de Dieu. Sartre versus Heidegger
}

\author{
Philippe Cabestan
}

À la mémoire de Michel Haar (1937-2003)

Et de Dominique Janicaud (1937-2002)

1 Alors que pendant des siècles, on s'est interrogé sur le mystère de la croix, de la résurrection et du jugement dernier, force est de reconnaître que ces questions, de nos jours, n'occupent plus guère les esprits. On en viendrait presque à se demander comment des chrétiens ont bien pu par le passé s'entretuer. Le Sacré-Cœur, La SainteChapelle, Notre-Dame de Paris sont désormais des monuments historiques - «les caveaux et les tombeaux de Dieu $»^{1}$ écrit Nietzsche - qui sont visités et photographiés chaque année par des millions de touristes, tout comme la muraille de Chine, le Rialto ou le temple d'Angkor Vat. Posons sans plus tarder notre question : que s'est-il passé ? Comment interpréter un tel événement ? En guise de réponse, on se contente parfois de reprendre l'affirmation du dément ou forcené (der tolle Mensch) : « Dieu est mort »². De fait, le commun des mortels semble de nos jours ne pas plus croire en l'existence de Dieu qu'en celle des dieux de l'Antiquité alors même que celle-ci comme celle-là passaient en leur temps pour évidente - même s'il convient de ne pas surestimer cette évidence ${ }^{3}$.

2 Pourtant, dire que Dieu est mort est loin d'être satisfaisant tant l'affirmation est équivoque. L'événement est, par excellence, l'objet de ce que Paul Ricœur dénomme le conflit des interprétations. L'une d'entre elles semble particulièrement prégnante. En effet, quelque soixante ans avant la publication du Gai savoir (1882), Auguste Comte prophétise la mort de Dieu4. Conformément à la loi de l'humanité ou loi des trois états, il annonce le triomphe inéluctable de l'esprit positif, c'est-à-dire de l'esprit scientifique sur l'esprit métaphysique et théolo-

gique. Autrement dit, l'esprit positif est appelé à délivrer l'humanité des chimères de l'imagination, propres à son enfance, de sorte qu'un jour animisme, polythéisme et monothéisme ne seront plus qu'un vague souvenir, à quoi une religion nouvelle est appelée à se substituer : la religion positive de l'humanité ${ }^{\text {. }}$ 
3 A vrai dire, si nous évoquons cette interprétation de la mort de Dieu, c'est parce qu'elle nous paraît ne pas devoir retenir longtemps notre attention. D'une part, loin de s'appuyer sur des travaux d'historiens qui lui donneraient une ombre de légitimité positive, elle présente un caractère éminemment spéculatif qui fait bon marché des données de l'histoire. D'autre part, si la philosophie des Lumières nous a accoutumé à parler de la religion, en croyant découvrir derrières les différentes religions de l'humanité une seule et même religion naturelle, il convient sans doute de manier ce concept avec précaution et, en historien rigoureux, de ne pas confondre des phénomènes spirituels très différents les uns des autres dont on persiste à ignorer l'hétérogénéité et la singularité lorsqu'on se contente de les ranger sous les catégories de l'animisme, du polythéisme et du monothéisme. Bref, la religion est sans doute une abstraction commode mais dangereuse et féconde en faux problèmes.

Aussi voudrions-nous dans les pages qui suivent nous situer dans une tradition bien différente et considérer la manière pour ainsi dire antithétique dont deux figures majeures de l'histoire de la phénoménologie envisagent à leur tour la mort du Dieu. Nous pensons à Martin Heidegger et à son essai : «Le mot de Nietzsche: Dieu est mort $»^{6}$. Mais aussi et tout d'abord à Jean-Paul Sartre, phénoménologue résolument athée. Or, l'un comme l'autre, n'ont-ils pas à leur manière ignorer l'événement même de la mort du Dieu chrétien?

\section{Sartre, l'incrédule}

5 Quoiqu'appartenant au siècle dernier, le cas de Jean-Paul Sartre nous paraît à bien des égards significatif de notre époque. Né en 1905, l'année même de la loi dite de séparation de l'Eglise et de l'État, Sartre grandit dans un milieu chrétien : son grandpère Schweitzer est protestant, sa grand-mère catholique et sa mère est élevée chez les sœurs ${ }^{7}$. Le dimanche, Sartre accompagne parfois sa mère et sa grand-mère à la messe. Mais en vérité, ni l'une ni l'autre ne sont vraiment pratiquantes. Simplement, comme Sartre l'écrit, « la foi des autres les dispose à l'extase musicale; elles croient en Dieu le temps de goûter une toccata $»^{8}$. Dans Les Mots, Sartre rapporte comment, sans crier gare, à l'âge de 12 ans, il perdit le peu de foi qu'il avait: «Un matin, en 1917, à La Rochelle, j'attendais des camarades qui devaient m'accompagner au lycée ; ils tardaient, bientôt je ne sus plus qu'inventer pour me distraire et je décidai de penser au ToutPuissant. A l'instant il dégringola dans l'azur et disparut sans donner d'explication : il n'existe pas, me dis-je avec un étonnement de politesse et je crus l'affaire réglée $"^{9}$. Ce qui est remarquable dans ce récit, c'est la manière dont l'idée de Dieu disparaît : sans pathos et sans explication. Autrement dit, l'idée que Dieu n'existe pas n'est pas une conclusion mais une "évidence" d'autant moins discutable et d'autant plus solide qu'elle est parfaitement irrationnelle et qu'elle s'impose à Sartre qui feint l'étonnement pour masquer son indifférence.

6 Dans ses entretiens avec Simone de Beauvoir, publiés après La Cérémonie des adieux, Sartre revient sur cet épisode en y ajoutant quelques détails. Surtout il revient sur la manière dont cette "révélation » s'est imposée à lui : «Je ne sais d'où est venue cette pensée, comment elle m'a frappé ; je me suis dit tout d'un coup : mais Dieu n'existe pas!». Quand Beauvoir, sceptique, le presse de retrouver « le travail qui a précédé cette intuition », Sartre en confirme la soudaineté même s'il la tient pour illusoire dans la mesure où elle est à ses yeux nécessairement le résultat d'un travail qui lui échappe 
pour l'essentiel ${ }^{10}$. De là ce qu'il appelle son athéisme idéaliste. Il s'agit alors d'un athéisme limité à l'abandon d'une idée sans que cet abandon affecte son rapport au monde. Simplement Dieu n'en fait plus partie. Ce n'est que des années plus tard, à la suite de conversations avec Nizan, que Sartre réalise, pour reprendre son expression, que «l'absence de Dieu devait se lire partout». En d'autres termes, Sartre passe progressivement d'un athéisme idéaliste à un athéisme matérialiste qu'il définit comme "l'univers vu sans Dieu $»^{11}$. Un tel athéisme n'a rien d'évident car il exige de l'athée une nouvelle conception de l'être qui ne peut être que le fruit d'une ascèse éradiquant méthodiquement tout reste ou vestige de religion dans son rapport au monde. Ainsi, il ne suffit pas de dire que Dieu n'existe pas pour en avoir totalement fini avec la religion et Sartre reconnaît qu'il est longtemps resté prisonnier de cette promesse de salut par l'écriture qui a été la grande affaire de sa vie et qu'il tient pour une forme de religion. En ce sens, comme il écrit, l'athéisme, alias l'assomption authentique de la contingence, " est une entreprise cruelle et de longue haleine ${ }^{12}$.

Ces premières considérations ne se rapportent toutefois qu'à la seule personne de Sartre, même si ce qui y est en question s'inscrit dans le contexte plus large d'une civilisation. Curieusement et sauf erreur de notre part, Sartre aborde rarement la mort de Dieu en tant que telle. On objectera peut-être que la mort de Dieu est au cœur de sa pièce, Le Diable et le bon Dieu, dont l'action se situe pendant la Guerre des Paysans allemands (1524-1526). On s'en souvient peut-être : après s'être épuisé à faire le Mal pour être immoral, puis à faire «le Bien pour être moral » ${ }^{13}$, Gœtz découvre que Dieu n'existe pas. Il déclare à Heinrich : «Heinrich, je vais te faire connaître une espièglerie considérable : Dieu n'existe pas. Il n'existe pas. Joie, pleurs de joie ! Alléluia $»^{14}$. Reste que cette prise de conscience, qui parodie la nuit du mémorial, demeure strictement individuelle et que Sartre ne lui donne pas la signification d'un événement historique. En outre, la problématique de la pièce est beaucoup plus morale que métaphysique ${ }^{15}$. Il en va de même lorsque, rappelant le "si Dieu n'existe pas, tout est permis " de Dostoïevski, Sartre en conclut qu'il n'y a pas d'exigences transcendantes et que l'homme est sans excuse. Autrement dit il ne peut pas se réfugier derrière une quelconque prescription morale qui s'imposerait à lui catégoriquement ${ }^{16}$. Si l'existentialisme sartrien prend acte de la mort de Dieu et place l'homme face à ses responsabilités, en revanche il semble ne pas se soucier de l'événement lui-même.

\section{Une relative indifférence}

Dans un article consacré en 1943 à L'Expérience intérieure de Georges Bataille, Sartre évoque bien la mort de Dieu, qu'il commente brièvement ainsi : "Dieu est mort: n'entendons pas par là qu'il n'existe pas, ni même qu'il n'existe plus. Il est mort : il nous parlait et il se tait, nous ne touchons plus que son cadavre $»^{17}$. Manifestement, Sartre se souvient de Nietzsche mais, contrairement au dément, l'événement ne "l'inquiète pas plus que ça”. Sans doute Sartre a-t-il conscience qu'il n'en va pas de même pour tout le monde. Dans son essai sur Mallarmé, Sartre tient les poètes de 1850 pour des « orphelins de Dieu» qui ont ressenti «le Grand Naufrage comme une mutilation " ${ }^{18}$. $\mathrm{Au}$ vingtième siècle, Bataille fait partie de ces hommes que Sartre appelle des survivants : comme Nietzsche, Heidegger ou Jaspers et à l'instar de l'homme moderne, Bataille serait tourmenté par ce silence du transcendant et la permanence de son besoin religieux. Dans les Cahiers pour une morale (1947), Sartre évoque à nouveau 
brièvement la mort de Dieu ${ }^{19}$. Il se contente alors de noter : «Le grand changement historique : la mort de Dieu, remplacement de l'éternel par l'infini temporel. Du temps de Dieu, l'homme était inessentiel par rapport à l'Eternel sans durée. Aujourd'hui Dieu est tombé dans le temps ${ }^{20}$. Comprenons que l'Histoire, l'idée du progrès infini, a pris le relais et s'est substituée à l'idée de Dieu.

Comment interpréter cette relative indifférence ? Une première explication peut être tirée de l'ontologie sartrienne et, en un sens, de l'immortalité divine. En effet, L'Être et le néant comme les Cahiers accordent une place toute particulière à l'idée de Dieu en tant que cause de soi ou Ens causa sui ${ }^{21}$. Il va de soi que ce Dieu n'est pas le Dieu d'Abraham, d'Isaac et de Jacob. Il correspond bien plutôt à cette idée de Dieu que Descartes fit sienne mais que saint Thomas refusait pour la simple raison que Dieu ne saurait se précéder ${ }^{22}$. Plus fondamentalement et par-delà l'histoire de la philosophie, cette idée d'un Dieu cause de soi est inscrite selon Sartre dans l'être de la réalité-humaine et trouve sa source dans le désir d'être Dieu qui hante le pour-soi. Dans L'Être et le néant, après avoir établi que le pour-soi est manque et que ce manque a pour objet l'impossible synthèse de l'en-soi-pour-soi, Sartre déclare : «Et qu'on ne nous reproche pas d'inventer à plaisir un être de cette espèce: lorsque cette totalité dont l'être et l'absence absolue sont hypostasiés comme transcendance par delà le monde [...], elle prend le nom de Dieu $»^{23}$.

10 L'homme est donc l'être qui projette d'être Dieu mais qui ne parvient qu'à réaliser un Dieu manqué2 ${ }^{24}$. Hypostase du désir d'être du pour-soi, Dieu cause de soi n'est pas une invention de la philosophie historiquement datée mais une idée anhistorique ou transhistorique, inscrite dans l'être de l'homme. C'est la raison pour laquelle, contre toute attente, Sartre envisage une description eidétique de Dieu ${ }^{25}$. L'entreprise peut dérouter : imagine-t-on une eidétique d'Athéna ou de Mercure? Mais dans le cas de Dieu le projet est loin d'être absurde, même si l'idée de Dieu est contradictoire et que Dieu n'existe pas. Ainsi, en conclusion d'une longue analyse de l'Ens causa sui, Sartre assimile l'être de Dieu à l'être de la conscience qui, parce qu'elle n'est pas une chose ensoi, a à être son propre néant : « Ainsi l'Ens causa sui est son propre néant. Mais néant de quoi ? Néant de fondement : il a la responsabilité d'avoir à être son être sans être le fondement de cet être [...]. Ainsi Dieu est un homme inauthentique, lancé dans la tâche vaine de se fonder et qui ne peut se créer parce qu'il est déjà ${ }^{26}$.

Il y a une autre explication à cette indifférence relative de Sartre qui, étroitement dépendante de celle que nous venons d'exposer, réside dans sa conception de l'histoire à l'époque des Cahiers. En effet, Sartre y soutient que "Tout est à chaque époque " ${ }^{27}$. Autrement dit, contrairement à ce que soutient Hegel dans La Phénoménologie de l'esprit, l'histoire n'est pas une succession de figures de la conscience, qui trouverait son accomplissement dans le savoir absolu. Car même si la mauvaise foi lui dissimule certains aspects de sa condition, l'homme est d'emblée une « conscience absolue de sa condition». Par exemple, dès l'Antiquité, l'épicurisme développe une conception rationaliste et matérialiste qui correspond à cette forme de raison que Hegel projette bien plus tard dans l'Histoire sous le nom de raison observante. De manière analogue, si pour Aristote maître et esclave le sont par nature, pour Socrate, il y a « unité de l'Esprit chez le Maître et chez l'Esclave». Sartre en conclut qu'une "époque est toujours infiniment complexe parce que tout y est donné et qu'elle est plutôt l'accent mis sur certains aspects de la condition humaine ». De ce point de vue, l'idéal communiste est aussi ancien que le monde ${ }^{28}$ et «le christianisme devait exister dans le polythéisme 
comme rapport personnel de certains hommes à certains dieux. Et réciproquement le christianisme est polythéisme chez beaucoup (les saints et la Vierge) ${ }^{29}$. Chaque époque est ainsi une nébuleuse de possibilités contradictoires et se distingue, cependant, par l'accent mis sur certains aspects de la condition humaine. Nous comprenons alors que la mort de Dieu est toute relative dans la mesure où le monothéisme chrétien est une possibilité inscrite dans la condition humaine, qui, en tant que telle, ne peut relever d'un passé révolu.

12 Cette "conception" est toutefois relativement éphémère dans l'œuvre de Sartre. Développée après guerre, elle précède le tournant opéré au tout début des années cinquante en direction du marxisme. Dès lors, Sartre développe une philosophie de l'histoire qui, sans exclure nécessairement la précédente, se veut rigoureusement matérialiste. La compréhension de l'histoire repose sur une évidence dite indépassable, énoncée par Marx dans Le Capital, selon laquelle «le mode de production de la vie matérielle domine en général le développement de la vie sociale, politique et intellectuelle $\star^{30}$. De ce point de vue, la mort de Dieu comme le christianisme ou la réforme protestante sont des événements spirituels dérivés qui, au même titre que $L a$ Déclaration des droits de l'homme et du citoyen, dépendent de l'évolution des conditions matérielles de l'existence sociale et de la lutte des classes. Irions-nous jusqu'à dire qu'il s'agit d'un non événement? Ce serait sans doute aller trop loin et donner dans une théorie du reflet que Sartre rejette résolument dans la mesure où il reconnaît à la conscience une certaine autonomie ${ }^{31}$. Mais on peut penser que Sartre, faisant grosso modo sienne la conception marxiste de la religion, est conduit à négliger l'événement de la mort de Dieu en tant que tel. En outre, parce que la religion, comme l'écrit Beauvoir en 1954, aurait longtemps servi d'idéologie à la classe dominante, la mort de Dieu est dans ces conditions plutôt une heureuse nouvelle ${ }^{32}$. Le matérialisme sartrien rejoint à sa manière le positivisme d'Auguste Comte dont il partage la foi sinon dans l'ordre du moins dans le progrès de l'humanité33.

\section{Un athéisme méthodologique}

On devine déjà le fossé voire l'abîme qui sépare Heidegger de Sartre. Il convient toutefois de rappeler que, pour Heidegger comme pour Husserl, la philosophie ne saurait se confondre avec la foi et que l'un comme l'autre professent un athéisme méthodologique. En effet, juif converti au protestantisme et se définissant comme un "protestant non dogmatique », Husserl subordonne la phénoménologie à la mise entre parenthèse de tout objet transcendant, $\mathrm{y}$ compris Dieu, quand bien même la transcendance de Dieu serait distincte de celle du monde ${ }^{34}$. Ses lecteurs savent néanmoins que la figure de Dieu réapparaît, à partir des années vingt dans des manuscrits demeurés longtemps inédits ainsi que dans sa conférence de Vienne $(1935)^{35}$. Husserl y distingue entre les dieux au pluriel, les puissances mythiques qui appartiennent au monde environnant et le concept de Dieu au singulier, porteur du Logos absolu. Il s'agit alors d'une nouvelle figure de Dieu, qui doit obéir aux lois eidétiques, et qui s'annonce dans la sphère de l'immanence absolue ${ }^{36}$. En outre, les conversations de Husserl à partir de 1931 avec la sœur bénédictine Adelgundis Jaegerschmid, confirment au besoin la profondeur des convictions religieuses de Husserl. Ainsi, en 1935, Husserl lui déclare-t-il : «La vie d'un être humain n'est rien d'autre qu'un chemin vers Dieu. J'ai essayé d'atteindre ce but sans l'aide de la théologie, 
de ses preuves ni de ses méthodes ». Et il ajoute un peu plus bas : «Je sais que ma façon de procéder pourrait être dangereuse pour moi si je n'étais pas un homme profondément lié à Dieu et croyant au Christ ${ }^{37}$.

Heidegger, pour sa part, naît dans un milieu catholique à Messkirch et se destine initialement à la prêtrise. C'est en 1911, à l'âge de 22 ans, qu'il interrompt ses études de théologie pour se consacrer à la philosophie. Pour autant, il ne saurait être question pour Heidegger de confondre l'une et l'autre dans la mesure où, comme il l'écrit en 1922, la philosophie est fondamentalement athée. Affirmation qu'il réitère en 1925 en précisant que "c'est justement dans cet athéisme qu'elle (la phénoménologie) devient, comme quelqu'un de grand l'a dit un jour, un "gai savoir" " ${ }^{38}$. A l'époque d'Être et temps, Heidegger prône donc un athéisme méthodologique qui exige, comme il le rappelle dans sa conférence de 1928, une séparation rigoureuse de la philosophie et de la théologie ${ }^{39}$. Cependant, il convient de ne pas se méprendre sur cet athéisme méthodologique. La règle, tout comme l'adjectif athée, souffre d'une certaine ambiguïté. Elle ne signifie pas que, pour le philosophe, Dieu n'existe pas. L'athéisme n'est pas, en l'occurrence, la négation de Dieu mais seulement un principe méthodologique qui permet à la phénoménologie de ne pas se tromper sur elle-même et sur la tâche spécifique qui lui incombe. C'est au nom de ce nécessaire partage que Heidegger tient le concept de phénoménologie catholique pour encore "plus inepte que celui de mathématiques protestantes $»^{40}$.

Dans sa conférence de 1928, Heidegger précise rigoureusement les places respectives de la phénoménologie et de la théologie ainsi que la relation de dépendance unilatérale de la théologie en tant que science positive vis-à-vis de la phénoménologie en tant que science première ${ }^{41}$. En effet, la théologie chrétienne - qui ne doit pas être confondue avec la théologie philosophique qui relève de l'onto-théo-logie - est selon Heidegger une science positive qui a comme toute science positive un prédonné qui est dans son cas la christianité (Christlichkeit) ou l'événement de la croix ${ }^{42}$. Heidegger écrit à ce propos : « l'étant qui est primairement révélé à la foi et à elle seule et ce qui en tant que Révélation temporalise tout d'abord la foi, c'est pour la foi "chrétienne" le Christ, le Dieu crucifié ». En d'autres termes, la théologie n'a d'autre objet que l'événement historique du sacrifice du Christ tel qu'il se donne à travers la foi. Mais la théologie en tant que science positive de la foi n'en est pas moins dépendante de la phénoménologie au sens où, d'une manière générale, toute interprétation ontique adéquate repose nécessairement sur un fondement ontologique. Ainsi la notion théologique de péché, par exemple, se comprend à partir de l'être du Dasein et plus spécifiquement à partir de l'existential de la faute (Schuld) et de l'être en faute (Schuldigsein) ${ }^{43}$. La reconnaissance de cette dépendance n'implique aucune confusion et, si la religion est « une possibilité fondamentale de l'existence humaine », elle n'en est pas moins pour Heidegger « d'un type tout à fait différent de la philosophie $»^{44}$.

\section{Le différend (Auseinandersetzung) avec Nietzsche ${ }^{45}$}

16 Cette articulation de la phénoménologie et de la théologie fait l'objet au cours des années trente d'une réélaboration qui doit être ressaisie à la lumière du " tournant ». A proprement parler, il ne saurait être ici question d'un tournant théologique, au sens où Heidegger renouerait d'une manière ou d'une autre avec le christianisme ${ }^{46}$. C'est à cette époque en effet que, interrogeant l'essence onto-théologique de la philosophie, 
Heidegger se demande ce que serait, par suite, une compréhension authentique du divin, c'est-à-dire le Dieu divin ou Déité (Gottheit). Avec ce terme repris de maître Eckhart, Heidegger distingue entre, d'une part, le Dieu chrétien, le Tout-Puissant, l'étant suprême, créateur du ciel et de la terre et, d'autre part, la Déité ou dieu des poètes ${ }^{47}$. Ce dieu est alors une des quatre dimensions (Geviert) du monde compris comme unité du ciel et de la terre, des mortels et des dieux ${ }^{48}$. Et c'est naturellement à ce dieu dont l'absence ou la fuite caractérise ces temps de détresse que sont les temps modernes, que se rapporte Heidegger quand, reprenant à sa manière le thème de la mort de Dieu, il déclare que notre temps "est marqué d'un double manque et d'une double négation: le "ne plus" des dieux enfuis et le "pas encore' du dieu qui va venir $»^{49}$.

L'affinité avec Nietzsche, « l'adversaire le plus intime », est ici manifeste ${ }^{50}$. Il n'est guère étonnant à cet égard que Heidegger, entre 1936 et 1940, consacre cinq semestres de cours à Nietzsche afin, comme il l'écrit, d'élucider son différend avec le dernier philosophe allemand qui, loin de tout athéisme vulgaire, a cherché «Dieu avec passion $»^{51}$. Et c'est à partir de ses cours sur Nietzsche qu'il rédige son essai intitulé : "Le mot de Nietzsche "Dieu est mort" " au début duquel il cite in extenso le paragraphe 125 du Gai savoir ${ }^{52}$. L'ambition de ce texte est non pas simplement d'éclaircir mais de comprendre " autrement » la pensée de Nietzsche et d'en saisir "l'impensé » à partir de sa place dans l'histoire de la métaphysique occidentale ${ }^{53}$. De ce point de vue, il faut certes, dans un premier temps, lire le mot de Nietzsche tel que Nietzsche lui-même le comprend, c'est-à-dire comme le triomphe du nihilisme et la disparition corrélative de l'opposition platonicienne du sensible et de l'intelligible. C'est ce que Heidegger dénomme "le sens métaphysique du mot pensé métaphysiquement: "Dieu est mort" $\aleph^{54}$. Mais tel n'est pas le sens ultime, selon Heidegger, de ce mot de Nietzsche, dont le dévoilement implique d'interroger à nouveau l'essence de la métaphysique.

En effet, loin d'être parvenu à dépasser la métaphysique alias le platonisme, l'œuvre de Nietzsche relèverait encore et toujours de la métaphysique tout en en ignorant l'essence ${ }^{55}$. Elle en constituerait même son stade terminal : non seulement en assimilant l'être à la volonté de puissance, elle participe à l'oubli de l'être qui est l'essence de la métaphysique mais, en outre, elle pousse la métaphysique à son achèvement, au sens où le suprasensible une fois discrédité, la métaphysique n'a plus d'autre possibilité de déploiement. C'est pourquoi la pensée de Nietzsche est pour Heidegger le véritable accomplissement du nihilisme ou encore l'expérience nihiliste du nihilisme, au sens où de l'être dans la pensée de Nietzsche, "il n'en est rien ${ }^{56}$. A l'interprétation nietzschéenne du nihilisme à partir de la volonté de puissance, Heidegger oppose une pensée du nihilisme à partir de l'être et de l'oubli de l'être. Il lui est dès lors possible d'identifier parmi les meurtriers de Dieu et du monde suprasensible, ceux qui leur ont asséné les coups les plus rudes. Il s'agit non pas de " ceux qui ne croyaient pas en Dieu » mais, paradoxalement, des croyants et de leurs théologiens qui ravalent Dieu au rang de valeur suprême : «Le coup le plus rude contre Dieu n'est pas que Dieu soit tenu pour inconnaissable, que l'existence de Dieu soit démontrée indémontrable, mais que le Dieu tenu pour réel soit érigé en valeur suprême $»^{57}$. Comprenons bien alors ce qui sépare désormais Heidegger de Nietzsche qui, via sa métaphysique de la volonté de puissance et l'assimilation de l'être à la valeur, apparaît comme l'ultime meurtrier de Dieu. C'est que la mort de Dieu ne se résume pas à l'incrédulité triomphante vis-à-vis du Dieu chrétien ni à la dissolution des idéaux du monde suprasensible mais renvoie désormais 
au nihilisme dans son sens heideggérien de sorte que le meurtre dont nous sommes tous responsables et que nous accomplissons sans y prêter attention est « le meurtre de l'être de l'étant $»^{58}$.

\section{Conclusion}

19 Pour une raison que l'on ignore, Sartre rend visite en 1952 à Heidegger. Sans surprise, la discussion entre les deux hommes tourne court et se transforme bientôt en un monologue de Sartre sur la nécessité de l'engagement. A la fin, confie Sartre à Jean Cau, "je parlais à son chapeau: un chapeau vert de chasseur de chamois " ${ }^{59}$. Tenter de concilier les points de vue de Sartre et de Heidegger sur la mort de Dieu serait une gageure dépourvue de sens. Un dialogue est-il même pensable? A coup sûr, Sartre est pour Heidegger de ceux qui, comme il l'écrit au terme de son essai sur Nietzsche, sont devenus incapables de chercher Dieu, des «voyous publics qui ont aboli la pensée et mis à sa place le bavardage $»^{60}$. L'attente heideggérienne du dernier Dieu comme l'affirmation - intempestive s'il en est - que "seul un dieu peut encore nous sauver " $^{61}$ sont, il est vrai, inaudibles pour un philosophe athée comme Sartre, habité par cette conviction proprement prométhéenne que ce sont les hommes qui font eux-mêmes leur histoire (dans un milieu donné qui les conditionne) ${ }^{62}$. Pour Sartre comme pour La Fontaine, «aide-toi et le ciel t'aidera ». Reconnaissons-en outre que, lorsqu'il s'agit de la mort de Dieu et, plus généralement, de ce qu'on appelle la religion, la réflexion sartrienne est un peu courte. Comment ne pas regretter que son athéisme comme son matérialisme le conduisent finalement à ignorer un événement dont l'importance, au regard de la modernité, est non moins essentielle que la révolution démocratique (Tocqueville) ou la physique galiléenne qui lui sont, semble-t-il, étroitement liées. Or, si Sartre n'en dit pas grand chose, c'est pour la simple raison que l'événement ne lui paraît nullement décisif et, par suite, ne mérite pas son attention.

L'interprétation heideggérienne du mot de Nietzsche, de son côté, ne laisse pas non plus d'être déconcertante mais d'une tout autre manière. D'une part, même si Heidegger persiste à inscrire son propre cheminement dans un cadre phénoménologique, au nom de ce qu'il appelle en 1973 une "phénoménologie de l'inapparent », on peut cependant avec Dominique Janicaud s'interroger sur la solidité du lien entre la démarche phénoménologique et la mise en question heideggérienne de l'essence de la métaphysique ${ }^{63}$. Nous avons vu que l'ambition de Heidegger était de comprendre Nietzsche sinon mieux du moins autrement qu'il ne s'était lui-même compris et de dégager l'impensé du mot de Nietzsche ${ }^{64}$. Dans cette perspective, même si Nietzsche se prépare à accueillir une divinité à venir qu'il nomme provisoirement du nom de Dionysos ${ }^{65}$, il n'en participe pas moins à ce «meurtre de l'être de l'étant " qui est au cœur de la méditation heideggérienne. Toutefois, de quelle herméneutique relève une telle interprétation de l'impensé du mot de Nietzsche? Peut-on encore parler d'une herméneutique au sens d'une description et explicitation (Auslegung) de la chose même, pour reprendre les termes d'Être et temps? Manifestement, l'interprétation de Nietzsche relève d'une tout autre démarche herméneutique qui substitue à la chose même la parole comme demeure de l'être ${ }^{66}$.

21 On peut enfin se demander si cette interprétation heideggérienne du mot de Nietzsche fait droit à l'événement de la mort du Dieu chrétien. Heidegger interroge-t-il bien l'événement en son événementialité ou, si l'on préfère, en son sens événemential ${ }^{67}$ ? On 
pourrait reprocher à l'herméneutique heideggérienne d'avoir par avance gommé l'imprévisibilité, l'irréversibilité, la singularité en un mot l'historicité de l'événement qui est, en tant que tel, institution (Stiftung), ouverture de possibles, commencement ${ }^{68}$. L'interprétation du mot de Nietzsche en tant que forme ultime de la métaphysique comme l'attente d'un Dieu qui vienne nous sauver manifestent avant tout la résistance heideggérienne face à la modernité en tant que commencement d'un monde duquel non seulement le Dieu Chrétien mais la déité (Gottheit) - comme en témoigne l'athéisme sartrien - a irrémédiablement disparu. Or la mort de Dieu n'est-elle pas cet événement - nous serions presque tenté de dire cet archi-événement - historique en vertu duquel, pour le meilleur comme pour le pire, un monde radicalement nouveau surgit sur les ruines de l'ancien, un monde sans religion, sans dieu (Gottlos)?

\section{NOTES}

1. F. Nietzsche, Le Gai savoir, trad. par P. Wotling, Paris, GF Flammarion, 2020, §125, p. 178.

2. Ibid. p. 125.

3. "L'évidente connaissance que nous avons des dieux montre bien qu'ils existent ", Epicure, Lettre à Ménécée, trad. par P. Pénisson, Paris, Hatier, 2007, p. 6. Cette assertion, qui n’a rien n'avoir avec un athéisme masqué comme on l'a parfois soutenu, ne doit cependant pas occulter que l'athéisme proprement dit "était déjà assez répandu à l'époque » et même avant, comme en témoigne Platon dans Les Lois. Dans Les Cavaliers, Aristophane se fait l'écho d'un certain scepticisme lorsque le premier serviteur demande au second: "Tu crois donc réellement aux dieux ? ». Platon, Les Lois, Livre X, trad. par L. Robin, Paris, Gallimard, bibliothèque de la Pléiade, 1950, p. 1002 et sq. ; Aristophane, Les Cavaliers, trad. par J.-M. Alfonsi, Paris, G.F. Flammarion, 2019, p. 50.

4. A. Comte, Plan des travaux scientifiques nécessaire pour réorganiser la société, Paris, AubierMontaigne, 1970. Ce texte expose pour la première fois, en 1822, la loi dite des trois états.

5. A. Comte, Système de politique positive, Paris, Vrin, 1994.

6. M. Heidegger, Chemins qui ne mènent nulle part (1949), "Le mot de Nietzsche "Dieu est mort" ", trad. par W. Brokmeier, Paris, Gallimard, 1962, p. 253-322.

7. A. Cohen-Solal, Sartre (1905-1980), Paris, Gallimard, coll. Folio Essai, 1985, p. 54.

8. J.-P. Sartre, Les Mots, Paris, Gallimard, coll. Folio, 1972, p. 25.

9. Ibid., p. 209.

10. S. de Beauvoir, La Cérémonie des adieux, suivi de Entretiens avec J.-P. Sartre, Paris, Gallimard, 1981, p. 610-611.

11. Ibid., p. 612-615.

12. J.-P. Sartre, Les Mots, op. cit., p. 211.

13. J.-P. Sartre, Cahiers pour une morale, Paris, Gallimard, 1983, p. 11.

14. J.-P. Sartre, Le Diable et le bon Dieu, Paris, Paris, Gallimard, collection Folio, 1951, p. 238.

15. M. Contat et M. Rybalka, Les Ecrits de Sartre. Chronologie, Bibliographie commentée, Paris, Gallimard, 1970, p. 235.

16. J.-P. Sartre, L'existentialisme est un humanisme, p. 36. Dans une perspective analogue, Sartre écrit : «Si Dieu n'existe pas, nous avons seuls à décider du sens de l'Être », Cahiers pour une morale, op. cit., p. 502. 
17. J.-P. Sartre, « Un nouveau mystique », Situations I, Paris, Gallimard, 1975, p. 186.

18. J.-P. Sartre, Mallarmé. La lucidité et sa face d'ombre, Paris, Gallimard, 1986, p. 24.

19. Aux dires de certains spécialistes, Sartre aurait consacré à Nietzsche une longue étude (non retrouvée) dans le cadre de ses Cahiers pour une morale dont on retrouve un écho dans le Saint Genet. V. de Coorebyter, Sartre avant la phénoménologie, Bruxelles, Ousia, 2005, p. 202 ; J.-P. Sartre, Saint Genet, comédien et martyr, p.385-390. J.-F. Louette, Sartre contra Nietzsche, Grenoble, Presses universitaires de Grenoble, 1996.

20. J.-P. Sartre, Cahiers pour une morale, op. cit., p. 90.

21. Ibid., p. 537.

22. R. Descartes, Réponses aux premières objections, œuvres philosophiques, tome II, édition F. Alquié, Paris, Garnier, 1967, p. 527.

23. J.-P. Sartre, L'Être et le néant, Paris, Gallimard, 2008, p. 126.

24. Ibid., p. 612 et p. 671.

25. J.-P. Sartre, Cahiers pour une morale, op. cit., p. 485.

26. Ibid., p. 535-536. On pourrait ajouter à cette eidétique que Dieu est regard, hypostase de l'autre en tant que regard objectivant. C'est en ce sens qu'il faut comprendre cette définition : «La religion : hypostase de l'Autre qui nous transforme en objet absolu pour une liberté qui n'est plus jamais objet ", J.-P. Sartre, Cahiers pour une morale, op. cit., p.23. Quoique Sartre ne s'y réfère jamais explicitement, cette essence ontologico-anthropologique de l'idée de Dieu rappelle la thèse de Ludwig Feuerbach qui assimile Dieu à l'objet de la conscience : «Dieu est l'intériorité manifeste, le soi exprimé de l'homme ». L. Feuerbach, L'Essence du christianisme, trad. par J.-P. Osier, Paris, François Maspero, 1968, p. 129-130.

27. Ibid., p. 98.

28. Dans L'Assemblée des femmes, Praxagora propose que «tous mettent en commun leurs biens, aient part à ceux de tous et vivent du même fonds commun ». Aristophane, L'Assemblée des femmes, trad. par M.-J. Alfonsi, Paris, Garnier Flammarion, 2019, p. 204, vers 716.

29. J.-P. Sartre, Cahiers pour une morale, op., cit., p. 98.

30. J.-P. Sartre, Questions de méthode, Paris, Gallimard, 1967, p. 47-48.

31. Sartre tient la théorie du reflet pour pré-marxiste. En d'autres termes, tout en reconnaissant à la théorie du reflet le mérite de situer maladroitement la connaissance dans le monde, Sartre lui reproche d'ignorer la négativité de la pensée ou plutôt de la conscience qui dévoile l'objet. J.P. Sarre, Critique de la raison dialectique, t.1, Paris, Gallimard, 1985, p. 37 note 1 et p. 149.

32. «Pendant longtemps la religion a tenu lieu d'idéologie aux privilégiés » écrit S. de Beauvoir qui, toutefois, quelques lignes plus bas, nuance son propos en reconnaissant l'ambiguïté du christianisme : certains chrétiens «nient que Dieu soit à la solde des puissants de ce monde » et conclut son paragraphe ainsi : « le fait est que la cote de Dieu a beaucoup baissé ». S. de Beauvoir, «La pensée de droite, aujourd'hui ». Publié dans Les Temps modernes en 1954, cet essai est repris dans un recueil intitulé Faut-il brûler Sade, Paris, Gallimard, 1972, p. 112. Notons que Sartre, en 1952, rapporte la mort de Dieu au triomphe de la bourgeoisie dont l'athéisme fait "partie intégrante de son idéologie ». Il s'agit cependant d'un "parricide involontaire» dont la bourgeoisie est elle-même effrayée car il se pourrait bien que « Dieu entraîne ses fossoyeurs dans la tombe qu'ils ont creusée pour lui ", J.-P. Sartre, Mallarmé. La lucidité et sa face d'ombre, op. cit., p. 16-17.

33. « Il faut croire au progrès. Et c'est peut-être une de mes dernières naïvetés ", J.-P. Sartre et B. Lévy, L'espoir maintenant, Lagrasse, Verdier, 1991, p. 30.

34. E. Husserl, Idées directrices, trad. par J.-F. Lavigne, Paris, Gallimard, 2018, § 58, p. 177.

35. E. Husserl, «La Crise de l'humanité européenne et la philosophie », La Crise des sciences européennes et la phénoménologie transcendantale, trad. par G. Granel, Paris, Gallimard, 1976, p. 370. 
36. E. Husserl, «Attitude intérieure de la prière et orientation intérieure phénoménologique », Grenzproblem der Phänomenologie, Husserliana, vol. 42, appendice 25 (texte traduit dans ce numéro de la revue, $c f$. infra).

37. A. Jaegerschmid, « Gespräche mit Edmund Husserl 1931-1936», Stimmen der Zeit, 199 (1981), p. 56, «Conversations avec Husserl », texte traduit par Julien Farges dans ce même numéro de la revue, cf. infra ; F. Dastur, «Le "Dieu extrême" de la phénoménologie. Husserl et Heidegger ", La phénoménologie en question, Paris, Vrin, 2004, p. 247.

38. M. Heidegger, Interprétations phénoménologiques en vue d'Aristote, trad. par P. Arjakovsky et D. Panis, Paris, Gallimard, 2016 ; M. Heidegger, Prolégomènes à l'histoire du concept de temps, trad. par A. Boutot, Paris, Gallimard, 2006, p. 126.

39. "Phénoménologie et théologie " in E. Cassirer/M. Heidegger, Débat sur le kantisme et la philosophie, trad. par P. Aubenque, Paris, Beauchesne, 1972, p. 119-120. J. Greisch, «Interlude: phénoménologie et théologie », Ontologie et temporalité. Esquisse d'une interprétation intégrale de Sein und Zeit, Paris, PUF, Epiméthée, 1994, p. 427 et sq.

40. M. Heidegger, Les Problèmes fondamentaux de la phénoménologie, trad. par J.-F. Courtine, Paris, Gallimard, 1985, p. 39.

41. J. Greisch, Ontologie et temporalité, op. cit., p. 436 ; F. Dastur, Heidegger et la pensée à venir, Paris, Vrin, 2011, p. 144-145.

42. Cette distinction est reprise dans «Le mot de Nietzsche "Dieu est mort" " où Heidegger oppose le christianisme qui est « la manifestation historique, séculière et politique de l'Eglise » et la christianité de la foi néo-testamentaire qui correspond à « ce qui est chrétien », M. Heidegger, Chemins qui ne mènent nulle part, op. cit., p. 265.

43. M. Heidegger, Etre et temps, trad. par F. Vezin, Paris, Gallimard, 1986, § 58, p. 337-346 ; M. Heidegger, « Phénoménologie et théologie », op. cit., p. 117.

44. Lettre à Elisabeth Blochmann, 8 août 1928, citée par Jean Greisch, Ontologie et temporalité, op. cit., p.432-433.

45. Nous reprenons la traduction de ce terme proposée par Michel Haar dans La Fracture de l'histoire. Douze essais sur Heidegger, Grenoble, Millon, 1994, p. 190.

46. On se souvient de la formule de Dominique Janicaud: «Sans la Kehre de Heidegger, point de tournant théologique ». Toutefois, ce tournant théologique n'est nullement le fait de Heidegger mais de ceux que Janicaud dénomme « les candidats à l'héritage théologique », comme Emmanuel Levinas, Michel Henry ou Jean-Luc Marion. D. Janicaud, Le Tournant théologique de la phénoménologie française, Combas, éditions de l'éclat, 1991, p. 19 ; F. Dastur, Heidegger et la pensée à venir, "Théologie et philosophie », op. cit., p. 150, note 4.

47. Dans les Beiträge zur Philosophie (1936-1938), Heidegger envisage le «Dieu à l'extrême » ou "dieu ultime» (der letzte Gott) comme un dieu tout autre qui n'est attendu que par le petit nombre de ceux qui sont tournés vers l'avenir et au premier rang desquels se tient le poète Hölderlin. M. Heidegger, «Le Dieu à l'extrême ", Apports à la philosophie. De l'avenance, trad. par F. Fédier, Paris, Gallimard, 2013, §256, p. 466-475.

48. "Plus sacré encore que tout Dieu est dès lors le monde, que la Bible au contraire réduisait à une créature divine », J. Beaufret, Dialogue avec Heidegger, Paris, éditions de Minuit, 1985, tome 4, p. 48.

49. M. Heidegger, Approche de Hölderlin, trad. par H. Corbin et alia, Paris, Gallimard, 1996. C. Dubois, Heidegger. Introduction à une lecture, Paris, Éditions du Seuil, 2000, p. 333.

50. M. Haar, La fracture de l'histoire, op. cit., p. 189 ; M. Haar, Nietzsche et la métaphysique, Paris, Gallimard, 1993, p. 211.

51. De ce point de vue, le dément (der tolle Mensch), « qui s'était mis à courir sur la place publique en criant sans cesse : "Je cherche Dieu", "je cherche Dieu" ", est Nietzsche lui-même, penseur du de profundis. F. F. Nietzsche, Gai Savoir, \$125; M. Heidegger, «Discours de rectorat», Ecrits 
politiques, trad. par F. Fédier, Paris, Gallimard, 1995, p. 103 ; «Le mot de Nietzche : "Dieu est mort" ", p. 322.

52. M. Heidegger, Nietzsche, trad. par P. Klossowski, Paris, Gallimard, 1971, T. I et T. II.

53. M. Heidegger, « Le mot de Nietzsche "Dieu est mort” ", op.cit., p. 258.

54. Ibid., p. 307.

55. M. Heidegger, "Dépassement de la métaphysique », Essais et conférence, trad. par A. Préau, Paris, Gallimard, 1958, p. 91.

56. M. Heidegger, « Le mot de Nietzsche "Dieu est mort" ", op.cit., p. 312.

57. Ibid., p. 313.

58. Ibid., p. 321. Il convient cependant de souligner que, de 1935 à 1952, de L'introduction à la métaphysique à Qu'appelle-t-on penser ?, la position de Heidegger à l'égard de Nietzsche a évolué et que nous privilégions ici l'essai de 1943, repris dans Chemins qui ne mènent nulle part. Cf. M. Haar, La Fracture de l'histoire, op. cit., p. 199.

59. J. Cau, «Croquis de mémoire », Les Temps modernes, N531-533, oct.-déc. 1990, p. 1127-1128.

60. M. Heidegger, « Le mot de Nietzsche "Dieu est mort” ", op.cit., p. 322.

61. «Mené en 1966, l'interview fut, conformément à la volonté de Heidegger, publié en 1976. M. Heidegger, Ecrits politiques. 1933-1966, trad. par F. Fédier, p. 260. Sur la réception de ce mot de Heidegger, cf. P. Aubenque, Les Etudes philosophiques, juillet-août 1976, p. 271.

62. J.-P. Sartre, Questions de méthode, Paris, Gallimard, 1967, p. 45.

63. D. Janicaud, Le Tournant théologique de la phénoménologie française, op. cit., p.17-18. Pour Françoise Dastur, au contraire, ce lien est manifeste : «C'est parce que la phénoménologie se définit déjà chez Husserl comme une méthode, un chemin qui conduit à la Sache selbst, à la chose même qui est en question, que Heidegger continue de définir en 1973 sa pensée comme une "phénoménologie de l'inapparent" ", F. Dastur, "La pensée à venir: une phénoménologie de l'inapparent?», Heidegger et la pensée à venir, Paris, Vrin, 2011, p. 20.

64. Sur «la stratégie heideggérienne de lecture» de Nietzsche, cf. M. Haar, La fracture de l'histoire, op. cit., p. 194.

65. Car Dionysos n'est qu'un nom d'attente et nullement le Nom enfin définitif et ultime du dieu. Cf. M. Haar, Nietzsche et la métaphysique, op. cit., p. 211.

66. Cette tension entre deux modalités de la démarche herméneutique est présente dès $\hat{E}$ tre et temps où Heidegger passe de la question du sens de l'être à la question du sens d' " être ", c'est-àdire du verbe être, C. Romano, "La Phénoménologie en tant qu'herméneutique », Au cœur de la raison, la phénoménologie, Paris, Gallimard, 2010, p. 890.

67. C. Romano, L'Événement et le monde, Paris, PUF, collection Epiméthée, 1998, p. 54-55 ; C. Serban, «L'Événement historique : un paradigme de la phénoménalité », Revue de phénoménologie ALTER, Paris, $\mathrm{N}^{\circ} 25,2017$, p. 150.

68. On pourrait adresser le même reproche à Jean-Luc Marion qui, en 1976, avançait cette «évidence »: «ce qu'on nomme, suivant la dernière (ou l'avant-dernière) parole métaphysicienne, la "mort de Dieu", ne signifie pas que Dieu passe hors-jeu, mais indique le visage moderne de son insistante et éternelle fidélité ». On ne saurait mieux dans une seule et même "évidence" faire droit et ne pas faire droit à l'histoire. J.-L. Marion, L'idole et la distance, Paris, Grasset, 1989, p. 11. 